\title{
PER ESEMPIO
}

\author{
Angela Condello ${ }^{1}$ \\ Nomina sunt consequentia rerum ${ }^{2}$
}

\section{RIASSUNTO}

Analogia, paradigma ed esemplarità condividono un'origine comune e suggestiva. Aristotele usa il termine "paradigma" per descrivere ciò che in seguito la tradizione filosofica ha chiamato "analogia". Questi tre dispositivi argomentativi sono da collocare nello spazio intermedio fra pratica e teoria nel diritto. In questo articolo discuto il fatto che si possa stabilire definitivamente se essi dimostrino la prevalenza della teoria sulla pratica o viceversa -il che richiama il dibattito diffuso nel contesto del realismo giuridico americano sulla questione se il diritto consista in esperienza (practice) o in logica. La tesi conclusiva é che sia pressoché impossibile stabilire l'origine della paradigmaticità.

Parole Chiave: Analogia. Dispositivi Argomentativi. Esemplarità. Paradigma.

\section{ANALOGO ED ESEMPLARE}

Perché scavare ancora nelle tracce dalla analogia, tema tutt'altro che ignoto alla dottrina giuridica, italiana ${ }^{3}$ e non solo? Perché, per farlo, è utile ricorrere ad una riflessione sulla esemplarità e sul suo ruolo nel diritto? Questo intervento è, tra l'altro, un tentativo di rispondere a queste due domande.

Anzitutto, mettiamo ordine. Analogia e paradigma: collocati in discorsi paralleli, talora tangenti, a tratti interferenti, ma originati diversamente, questi due dispositivi hanno una origine intrecciata e piuttosto suggestiva. Ciò che importa non è separarli e collocarli definitivamente, ma tentare di scoprire come sono comparsi nella storia del pensiero giuridico. Se gli esempi del loro uso nelle corti -in particolare di ultima istanza, ed anche costituzionali- sono moltissimi ${ }^{4}$, una loro collocazione sistematica ancora manca. La descrizione della loro Entwiklung come concetti e come dispositivi deve muovere dalla filosofia classica e, trasversalmente, arrivare al pensiero del XX secolo, mentre parallelamente è utile partire dagli usi che emergono dalla prassi per provare a porsi delle domande rilevanti.

Il luogo obbligato della archeologia del ragionamento analogico come paradeigma è la tradizione aristotelica ${ }^{5}$. Qui vengono distinte tre specie di inferenza: la deduttiva, che va dal generale al particolare; l'induttiva, che va dal particolare al generale; e l'analogica, che va dal particolare al particolare. Va detto che la definizione del terzo tipo di inferenza fa riferimento esplicito al paradeigma e non alla analogia. Siccome, però, la tradizione filosofica ha tradotto il paradigma aristotelico come inferenza analogica, il richiamo all'uno 
quando si discute dell'altro è inevitabile ${ }^{6}$. Sebbene la genesi sia dunque comune, il latino exemplum, corrispondente del greco paradeigma, si è sedimentato quasi esclusivamente nel campo della retorica come strumento di persuasione7; qui l'arte consiste nell'uso dell'entimema (ragionamento che omette una delle premesse, lasciandola sottointesa), ma anche nel risolvere mediante esemplificazione (o illustrazione paradigmatica) le difficoltà intorno alle tesi proposte.

La classificazione aristotelica delle forme di inferenza è condotta in base alla natura dei termini -generale, particolare- e non alla forma dell'argomento; secondo il filosofo italiano Enzo Melandri questo è l'errore di fondo nello schema sviluppato negli Analitica Priora. Nel passare da un particolare ad un altro, come ha sostenuto Aristotele, ci sono due possibili alternative. O si presuppone entimemicamente una premessa generale -come accade spesso nella giurisprudenza della Corte Europea di Giustizia, ad esempio, un principio generale $^{9}$-, e allora l'argomento è deduttivo; o la premessa generale viene stabilita in base al passaggio da un particolare ad un altro particolare, e allora l'argomento è induttivo. Quale dei due casi corrisponde alla analogia? Nessuno dei due. Che valore probativo ha, quindi, l'esempio? II valore della paradigmaticità, è presto detto; e questa tautologia non va sottovalutata.

Per un verso, ciò porterebbe a credere che esemplificando una legge generale l'esempio e l'uso che ne viene fatto la semplifichino ${ }^{10}$, come suggerisce l'espressione di un giurista francese "ce que fait que la comparaison peut divenire raison"11. Ma non è così, esemplificare non è semplificare, esattamente come comparare può servire a spiegare ${ }^{12}$, ma l'explicatio è ben altra cosa ${ }^{13}$. A quale modello si avvicina di più il pensiero analogico, all'induttivo 0 al deduttivo? Generalmente si ritiene che il ragionamento paradigmatico sia composto da una induzione e da una successiva deduzione. II che non è sbagliato, ma tende a mantenere il discorso su un piano strettamente logico; e la analogia, così come il suo complemento originario che è l'esempio, se osservata con la lente della logica e solo con quella, non è colta nella sua profondità. E' un dispositivo che si origina dalla prudenza ${ }^{14}$ delle pratiche oppure ha nel piano teorico il suo fondamento? Non è facile rispondere; o meglio: verosimilmente non è questa la domanda a cui rispondere. Infatti, se l'approccio tipico della logica è sintetizzabile nella binomio tutto-o-nulla, che significa bivalenza e principio del terzo escluso, uno sguardo più lungo sul paradeigma ci svela che il terzo nella esemplarità è incluso. Inclusione ed esclusione sono cifre con cui il diritto si misura quotidianamente; ogni dialettica legata al binomio universale/particolare, ad esempio, è costruita sulla scelta fra inclusione ed esclusione. Non solo, ma il diritto è un campo particolarmente interessante in questo senso, perché in esso risulta ancora più evidente la differenza fra la dualità di elementi semplici, come (all'apparenza possono sembrare) i casi e le loro fattispecie, e il principio di identità funzionale, per cui il singolo oggetto preso a sè non è definibile, se non in quanto valore che soddisfa alle funzioni svolte, dalle quali a sua volta dipende. Una decisione, infatti, è sempre parte di un contesto più largo. Analogo ed esemplare vanno quindi anzitutto osservati sotto questa lente. 


\section{SCHEMA, MODELLO, ARCHÉ ${ }^{15}$}

Torniamo dunque per un momento al paradeigma. Ciascun esempio è in funzione di qualcos'altro. II giudice talvolta usa una analogia per legittimare una decisione; in altri casi si richiama ad uno standard ${ }^{16}$ per supportare una linea teorica o per integrare il proprio ragionamento attraverso un modello $o$ uno schema - termine che, come è noto, va ricondotto al pensiero kantiano. L'esempio ha tanto più valore probativo quanto meglio riesce a rappresentare il generale di cui è il particolare. La legge, dal canto suo, deve comprendere una infinità di casi possibili.

L'esempio, se confrontato con la legge, rappresenta solo se stesso, e allo stesso tempo è ancora più complesso della legge, poiché rappresentando se stesso può comprendere anche altri casi. Esso è infatti non solo paradigma, ma anche caso particolare. Nella sua concretezza e pragmaticità raccoglie una lunga serie di determinazioni.

II ragionamento analogico, con la forza ${ }^{17}$ della esemplarità a cui ricorre ${ }^{18}$, in prima istanza mostra ${ }^{19}$. Mostrare significa illustrare, rappresentare ed indicare a riferimento; il rinvio ad un contesto esemplare è uno strumento pratico di cui il diritto si serve, spesso implicitamente: esso descrive regole quando la astrazione risulta troppo complessa, dunque ha in questo senso anche una funzione fortemente pedagogica ${ }^{20}$, nel senso che è come se

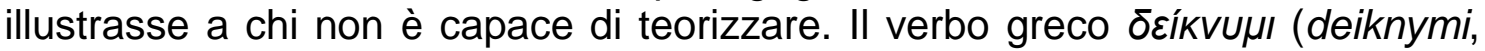
mostrare) significa anche indicare, per cui paradigmatico è ciò che "mostra accanto" (''accanto è il parà di $\pi \alpha \rho a ́ \delta \varepsilon I y \mu \alpha)$. II paradeigma ha una doppia natura: è l'immagine che rimanda ad un discorso -proprio come l'uso analogico di una fattispecie normativa o di una decisione precedente è insieme un fotogramma del processo applicativo-, ed insieme il ragionamento che legittima la scelta di una linea argomentativa rispetto ad un'altra. Talora fra i giuristi manca la consapevolezza di questo doppio legame.

Kant era assai meno inconsapevole; nella Metaphysik der Sitten ${ }^{21}$ usa infatti indifferentemente Exempel e Beispiel, poi specificando in una nota che "la parola tedesca Beispiel, che si adopera comunemente come sinonimo di Exempel, non ha però precisamente lo stesso significato. Prendere un Exempel e citare un Beispiel per la maggior chiarezza e comprensibilità di un'espressione, sono due concetti affatto diversi. L'Exempel è un caso particolare di una regola pratica, in quanto questa rappresenta un'azione come praticabile o impraticabile. Un Beispiel, all'opposto, è soltanto il particolare (concretum) rappresentato come contenuto nell'universale secondo concetti (abstractum), e l'esposizione puramente teorica di un concetto". E' una differenziazione fra uso e menzione, che consente proprio a partire dalla attenzione all'uso, alla pratica, di risalire dal singolare all'universale e, per lo stesso motivo, di ridiscendere nel verso opposto. Questa profonda ed inscindibile correlazione fra piano astratto e piano concreto è ciò che Christian Wolff osserva equiparando l'atto logico del giudizio ed il giudizio sensibile: dire 
infatti che un triangolo possiede tre lati è, al contempo, sia un atto logico sia un atto sensibile ${ }^{22}$.

Queste osservazioni si legano a ciò che voglio sostenere a proposito dell'uso di analogia ed esempio nel discorso giuridico: essi non sono solo logic, ma nemmeno solo experience ${ }^{23}$. I giuristi si sono intrattenuti a lungo nel dibattito intorno alla dicotomia prassi/teoria ${ }^{24}$, ma, come era prevedibile, uscirne con una risposta definitiva è risultato impossibile. Scavando ancora in Kant ${ }^{25}$ e in Wolff, possiamo invece tentare di restituire questo nodo al suo campo semantico di appartenenza. L'esempio non pare affatto un dato estrinseco al giudizio $^{26}$ : esso ne permette la particolarizzazione e può al contempo valere per dimostrare la realtà dei concetti. L'uso delle analogie nel ragionamento giuridico -sia esso euristico o meno- non è poi così distante (tutt'altro, anzi) dallo schema di Kant. II ricorso alla filosofia è oltremodo importante per comprendere il problema della origine delle analogie e la questione della loro validità al variare dei contesti. Infatti, l'esempio, assunto come regola operativa, è il precipitato concreto di un concetto -nel diritto, di un tema già noto, come fattispecie normativa o come precedent.

Questo discorso assume rilievo se si considera che il diritto ricorre con incredibile frequenza a comparazioni, poiché è nella sua natura ${ }^{27}$. Ogni confronto con un modello, uno standard, un caso $^{28}$ o una norma prevista per un altro caso, è la premessa che legittima una inferenza finalizzata alla decisione. A mio avviso, molte delle possibilità argomentative di cui il diritto si serve sono strutturate sul ragionamento analogico, che è per eccellenza inferenza a partire da un confronto. Esse formano una vera e propria topica della analogia e della paradigmaticità nel campo giuridico. Sono sospese fra lo spazio visibile e concreto e quello invisibile che riguarda il concreto, fra singolare ed universale $^{29}$, produzione e riproduzione ${ }^{30}$. Esemplare e schematico lavorano entrambi per trovare dei criteri congruenti per la generalizzazione ${ }^{31}$; essi sono due attributi interscambiabili se osservati dalla prospettiva del giurista, e richiamano la trasversalità fra prassi e teoria che è il filo rosso di questo discorso.

Se lo schema, kantianamente, è inteso come idea, esso fornisce la regola: è un Urbild che può determinare le possibilità del successivo Nachbild ${ }^{32}$. Ciò che caratterizza il rapporto fra piano intellegibile e piano sensibile, o fra determinato e generale, è la mediazione di un momento terzo, che funziona da connettore, e che in Kant è l'immagine. Ora, posto che ogni fenomeno giuridico che coinvolga il ragionamento analogico, il riferimento ad esempi, l'uso di modelli o standard, è dunque da collocare su una linea trasversale fra fatti e norme ${ }^{33}$, risulta più chiara la quaestio che è davvero rilevante in questo discorso. Mi riferisco al problema del paradigma come fenomeno originario.

Come l'Urphänomenon ${ }^{34}$ di Goethe, l'analogical reasoning -per dirla con i giuristi di Common Law- va alla ricerca del "fenomeno originale". Cosa si intende con questa espressione? II riferimento anteriore, sul piano temporale, su quello logico o su entrambi; la definizione che manca e che, per qualcosa di simile, esiste già, proprio come accade con le complessità già ridotte. 
Come il fenomeno originale, esso va inteso in senso strettamente paradigmatico. Ritorna la radice tedesca Ur-, che è significativa se associata all'idea di un'origine che determina e informa i fenomeni ad essa successivi, ed al contempo ne è informata. In questo senso l'analogia illustra come dal passaggio attraverso una descrizione già sviluppata, una soluzione già trovata, si arriva a toccare la struttura che informa l'ordinamento intero. Lo si comprende ancora meglio con un semplice esempio: nella gran parte dei casi, il giudice, ricorrendo al ragionamento analogico e servendosi di paradigmi, legittima la scelta secondo la maggiore rilevanza di una somiglianza rispetto ad un'altra; per farlo, ricostruisce la ratio, cha altro non è se non ciò che ha originato la norma e che continua ad informare e a caratterizzare il modo in cui questa agisce.

L'Urphänomenon goethiano, come l'Ursprung di Walter Benjamin ${ }^{35}$, rimanda alla semantica dell'archés6, di una origine che è sia principio che comando, che, nell'atto del dare forma, caratterizza. Discorso legato alla fenomenologia ${ }^{37}$, quello dell'origine di una analogia intesa come paradeigma. Come si determina, dunque, un'analogia?

\section{GENERALIZZARE E DEFINIRE}

II modello di "esperienza di specie superiore"38 di cui parla Goethe ha la capacità di unificare i singoli fenomeni; in esso ciascun precipitato, ciascun evento "si tiene in relazione con innumerevoli altri, nel modo in cui diciamo di un punto luminoso liberamente oscillante, che esso manda i suoi raggi in ogni direzione"39. L'esempio è quindi, come ha osservato Maurizio Ferraris, simultaneamente il caso singolo e l'indice di una classe, secondo una prestazione trasversale rispetto alle categorizzazioni dell'individuo, del genere e della specie ${ }^{40}$.

Nel legare il caso singolo ad un sistema più ampio in cui esso è inserito ed in funzione del quale deve essere deciso, si attraversa quel luogo che è stato definito la "esemplarità dell'esempio" ${ }^{41}$. Prendendo ad esempio qualcosa da una classe, selezioniamo determinate caratteristiche che lo differenziano e che lo rendono rappresentativo di quella determinata classe. Esattamente come fa il giudice quando, ricorrendo alla analogia, si richiama a criteri di rilevanza generalmente diffusi nel sistema in cui opera, per i quali una inferenza analogica può legittimamente fondare un ragionamento. Riconoscendo ad un caso il tenore di precedent, ad una fattispecie quello di simile, ad un contesto o elemento il valore di standard ${ }^{42}$, si riconoscono in essi caratteristiche che sono generalizzabili.

Il cuore della questione è dove e come inizi il percorso che conduce ad accettare una generalizzazione piuttosto che un'altra, che è lo stesso problema della natura originale dell'esempio, di cui si è detto poc'anzi. La cosa interessante a questo proposito è che, nella risalita verso la definizione che 
comprenda possibilità con caratteri comuni, non si ha a che fare con una operazione che, apriori, precede l'esemplare, ma con un giudizio formulabile soltanto a partire dal caso concreto. E' la stessa cosa che accade con l'opera d'arte, che può diventare "canone" 43 soltanto dopo essere apparsa nel mondo. Ancora una volta viene in aiuto Kant. Se è piuttosto difficile determinare dove si origini l'esemplarità di una parte rispetto ad una classe da essa rappresentata, si può dunque tentare un percorso differente; che le cose abbiano delle proprietà apriori o che tali proprietà siano "scoperte" 44 empiricamente, sono entrambe opzioni possibili, ma entrambe parziali. Allargando lo sguardo, si può osservare che le cose possiedono, per natura ${ }^{45}$, delle regolarità intrinseche (Ferraris le definisce "legalità intrinseche" ${ }^{46}$ ), che le rendono tipiche ${ }^{47}$, e ciò senza che le loro essenze siano conoscibili apriori. Quando nella Kritik der Urteilskraft $^{48}$ (§75, Der Begriff einer objektiven Zweckmäßigkeit der Natur ist ein kritisches prinzip der Vernunft für die reflektierende Urteilskraft) affianca al giudizio determinante il giudizio riflettente, Kant in verità supera il primo e gli sostituisce il secondo. Il giudizio determinante è il passaggio da una regola generale al caso, mentre quello riflettente è costruito sul passaggio dal caso al piano generale, anche se non necessariamente universale -poiché non è detto che in esso siano comprese tutte le possibilità.

Analogia è quindi, anzitutto, un dispositivo ermeneutico di cui il diritto si serve in circostanze complesse, prevalentemente in presenza di un elemento nuovo, non previsto, non calcolato, non ancora descritto. E' qui che l'esperienza del già deciso o del già descritto entra in azione, come risultato di un processo di concettualizzazione che ha condotto alla creazione artificiale di fatti-specie (termine che evoca la doppiezza concreto/astratto).

Un'indagine nella giurisprudenza di corti italiane ${ }^{49}$, della Supreme Court of England ${ }^{50}$ e della Supreme Court of the United States dimostra che i termini analogy ${ }^{51}$, analogical reasoning, similar circumstances, similar concept sono usati talora con obiettivi differenti. Questa è l'ulteriore conferma del fatto che, sebbene da un punto di vista logico la analogia non abbia ancora trovato una sistemazione rigorosa (ed è dubbio che mai possa trovarla), il diritto - come altri campi del sapere- ne fa uso costante. Non solo: gli esempi più pregnanti sono offerti dal diritto internazionale, dal diritto dell'Unione Europea, dalla lex mercatoria ${ }^{52}$; analogo ed esemplare abbondano laddove c'è carenza di definizioni. Qui entrano in azione, a colmare, ad integrare, a legittimare la decisione. I sistemi giuridici menzionati si distinguono da quelli nazionali per il carattere meno denso ed articolato del loro corpo normativo; per esempi e per analogie, si indaga un luogo inesplorato ${ }^{53}$, si crea una nuova relazione.

Qualcosa di molto simile avviene attraverso le forme di generalizzazione ${ }^{54}$; la storia del diritto dimostra che il pensiero giuridico vi ha fatto spesso ricorso per la soluzione di casi controversi. La costruzione di una fattispecie generale che valga per più casi è un processo complesso; esso si fonda su una definizione di tipo fenomenologico per cui l'esperienza (e non solo) viene tradotta in normatività. In un certo senso ciò richiama il tema luhmanniano della generalizzazione congruente delle aspettative. II processo di generalizzazione, che dal dettaglio del caso singolo ${ }^{55}$ conduce a decifrare 
l'attributo comune condiviso con altri casi, è fondamentale nel diritto. A questo proposito la semantica suggerita dai prefissi ci viene in soccorso.

Originariamente, nella lingua greca anà si riferiva alla "risalita" dal basso verso l'alto ed era l'opposto di katà (movimento verso il basso lungo un percorso) - ciò che avvicinerebbe la analogia più largamente ai processi di generalizzazione di cui si tratta. In seguito, dall'idea di risalita lungo una serie di elementi, intesa come spazio percorribile, è derivato l'uso distributivo della preposizione, attestato ad esempio in Senofonte, Anabasis ${ }^{56}$ (4, 6). Altrettanto interessante è la struttura della parola paradeigma, che si compone di parà e deiknymi: mostrare a fianco a qualcosa. II caso esemplare mostra infatti come dovrebbero essere gli altri, come si dovrebbe decidere, o almeno quale dovrebbe essere l'orientamento di riferimento. Tra gli usi figurati di parà troviamo pure alcuni che supportano piuttosto l'idea del "contrariamente a", "altrimenti che", come nell'uso che ne fa Eschilo nei Supplici (454). Spesso, il significato figurato è molto più vicino a quello spaziale di quanto si sarebbe portati a credere. Inoltre, il parallelismo con il senso del "lungo qualcosa" (che è proprio anche di anà) ha assicurato lo sviluppo delle semantiche "sotto la dipendenza di", come si osserva ad esempio in Isocrate, $(6,52)$, e "rispetto a", come nei Memorabili di Senofonte $(1,4)$.

\section{DOVE SI ORIGINA UN PARADEIGMA?}

Uno dei dilemmi ricorrenti intorno al ragionamento analogico nel discorso giuridico è legato al vecchio dibattito riassunto dalla affermazione di Oliver Wendell Holmes "the life of the law has not been logic, it has been experience" ${ }^{\prime 57}$. E' infatti nello scarto fra pratiche e logica che si snoda tutto il problema dello "stare al posto di una definizione" nel discorso giuridico. Come si è detto, secondo Enzo Melandri la ripartizione aristotelica dei tre tipi di inferenza contiene una contraddizione, che sta nel rilievo assegnato al tipo di termini coinvolti piuttosto che alle dinamiche ${ }^{58}$. Credo che questo sia il punto, d'altro canto, della incompletezza epistemologica del dibattito statunitense a proposito della rilevanza della ragione o delle pratiche nel diritto, per quanto riguarda la decisione dei casi. Mi riferisco alla nota domanda, diffusa fra i giuristi statunitensi alla fine del XIX secolo, se nel diritto rilevi più il livello pragmatico o quello definitorio; problema che, con buona pace di che credeva che non fosse più necessario ritornare a lavorarci, è riemerso negli ultimi anni ${ }^{59}$, in particolare a proposito della soluzione dei cosiddetti hard cases. Ronald Dworkin ${ }^{60}$, criticando il pragmatista Richard Rorty, troppo concentrato sulla realtà da aver trascurato, a suo avviso, la teoria, ha sostenuto che se si intende davvero rifiutare un resoconto del ragionamento nel diritto, si dovrebbero allora anche accantonare le pretese di integrità e coerenza di un ordinamento. La risposta di Cass Sustein, in linea con il pragmatismo suggerito da Edward Levi ${ }^{61}$, che a sua volta deve senz'altro qualcosa alla tradizione filosofica del pragmatismo americano di fine $1800^{62}$, è che il giudice debba decidere sugli hard cases non volgendosi a livelli più astratti di teoria, ma "più da giurista": con la analogia ${ }^{63}$. Al coro va aggiunto Richard Posner, che nel suo testo How Judges Think ${ }^{64}$ 
ricostruisce la storia del pragmatismo nel pensiero giuridico e in filosofia e tenta di tracciarne le connessioni e le divergenze. L'ideologia di Holmes ${ }^{65}$ rappresenta la posizione dominante del realismo giuridico degli anni '20 e '30 negli Stati Uniti, fra cui spiccano i nomi di Cardozo, Felix Cohen, Max Radin e Karl Llewellyn. II punto centrale di questa prospettiva era il carattere realistic del potere giudiziario e la affermazione dei limiti epistemologici del diritto. II cuore del pragmatismo giuridico è il problema della adjudication: essa è necessità e concretezza, e non generalizzazione o concettualizzazione ${ }^{66}$.

Ora, se la analogia sia indice della prevalenza delle pratiche e dell'esperienza sulla logica, nel diritto e negli altri campi del sapere, non è dato saperlo, a mio avviso. E una buona indagine, è cosa nota, deve perseguire interrogativi rilevanti.

Per quanto finora sostenuto, ritengo che sia ben più importante domandarsi: quando si origina (se si origina) una analogia? Dove si formano gli esempi? Come ha osservato Maurizio Ferraris ${ }^{67}$, exemplum è sia lo specime empirico sia il paradeigma; secondo la determinazione platonica, esso è prototipo (stessa natura di Ur) ed ectipo insieme, non l'uno senza l'altro. Le due prestazioni, prototipo ed ectipo, ciascuna con le proprie modalità, sono subordinate -è evidente- del typon. L'analogon, a sua volta, ha a che fare sia con il passato -un caso già deciso, una fattispecie già prevista- sia con il futuro, nella forma di una traccia per ciò che andrà detto, che andrà deciso. Per cui è logica e prassi insieme, e mostra più di ogni altro dispositivo della argomentazione giuridica che questi due piani non solo hanno punti di interferenza, ma sono profondamente relazionali e non vanno definiti separatamente.

\section{QUANDO È DEFINITIVA UNA DEFINIZIONE?}

Michel Foucault nel 1969 scriveva "en gènéral l'histoire des idées traite le champ des discours comme un domaine à deux valeurs; tout élément qu'on repère peut être caracterisé comme ancien ou nouveau; inédit ou répété; traditionnel ou original; conforme à un type moyen ou déviant. On peut donc distinguer deux catégories de formulations; celles, valorisées et relativement peu nombreuses, qui apparaissent pour la première fois, qui n'ont pas d'antécèdents semblables à elles, qui vont éventuellement servir de modèles aux autres, et qui dans cette mesure méritent de passer pour des créations; et celles, banales, quotidiennes, massives qui ne sont pas responsables d'elles mêmes et qui dérivent, parfois pour le répéter textuellement, de ce qui à été déjà dit ${ }^{168}$.

Ciascuno a modo proprio, esempio ed analogia, osservati come dispositivi che operano in una più vasta dinamica, suggeriscono una prima conclusione. Nel contesto giuridico, la logica -se intesa in senso stretto- non arriva ad esercitare alcuna egemonia sul pensiero razionale; inoltre, se essa è intesa in senso lato, deve comprendere e giustificare la frequenza con cui si ricorre tanto agli esempi quanto al ragionamento ed alla argomentazione 
analogici. Dunque, la logica deve fare i conti anche con la contraddizione insita alla sua stessa struttura ${ }^{69}$. Osservando questi strumenti all'opera, si ha conferma di ciò che aveva già rilevato Enzo Melandri nel suo lavoro sulla analogia fra logica e filosofia: "la morale della favola- diceva alla fine della sezione dedicata al calcolo analogico- la traiamo dalla filosofia del diritto"70. Gli usi della analogia, così come la forza della paradigmaticità ed il valore esemplificativo della prova, mostrano che "non c'è una scienza che per principio si sottragga a questa conclusione: la presenza di una tropologia (di un sistema di metafore, di costrutti teorici, \& c.) indica la necessità di un complemento allegorico-tropologico"71. I tropi, le figure, servono a colmare una mancanza, o a chiarire un dubbio attraverso il rimando ad un'immagine nota. Arricchiscono, aggiungono qualcosa e lo fanno, ogni volta, creando connessioni, siano esse attese o inattese. Tali connessioni sono il risultato di un trasporto: attraverso il rinvio, esse fanno scivolare le connotazioni dei concetti e le adattano secondo funzioni e necessità. I tropi, nel diritto e non solo, stanno al posto di una definizione, colmano con una descrizione ${ }^{72}$ senza luogo la necessità di mettere un punto al discorso; ciò nel diritto accade con nel meccanismo decisionale, che mostra il bisogno di pervenire ad un accordo in modo eloquente.

Osservando l'uso che del termine analogy viene fatto nel diritto, possiamo scomporre il ragionamento analogico ed distinguere in esso un "argomento", un "giudizio" e un "concetto". Un concetto è analogico quando non se ne può definire il significato in maniera univoca: i concetti analogici sono quindi campi "aperti"; il carattere della apertura è problema tipico della fenomenologia: le stesse caratteristiche dentro cornici differenti cambiano riferimenti $^{73}$. In questo senso, alcune norme o alcuni casi sono analogici se interpretati come concetti.

Il diritto è ricco di esempi in cui ad essere analogico è più esplicitamente un giudizio (nel senso generico di "valutazione", non come decisione di una corte); questo si distingue dal concetto analogico per il semplice fatto che, a differenza del concetto, il giudizio può essere detto vero o falso. II giudizio analogico, comparativo o proporzionale, è diverso dai giudizi ordinari, che tipicamente sono a carattere categorico. La logica sottostante un giudizio ordinario, non analogico, si basa sul principio del terzo escluso, vale a dire del "vero-o-falso", che è una logica bivalente. Nel giudizio analogico, al contrario, non solo il tertium non datur non regge, ma è molto più coerente fare riferimento al "terzo incluso". Perciò nella ana-logica si sostituisce "vero-o-falso" con "verosimile o più o meno vero e più o meno falso" 74 .

A proposito infine dell'argomento analogico, un'indagine nelle più recenti fonti giurisprudenziali, nazionali e internazionali, ha confermato la tesi per cui esso non sia propriamente un genere di argomento, ma piuttosto una intera famiglia di argomenti affini. Esso può infatti essere: ragionamento analogico, rinvio ad un esempio, a modelli, a definizioni tramite standard. Linguisticamente, inoltre, può essere: esempio, proporzione, interpretazione o analogia attributiva, entimema o argomentazione retorica, uno qualunque fra $\mathrm{i}$ tropi induttivi, uno qualunque fra i tropi retorici. Quindi si può dire, per riordinare il rapporto fra analogia e paradeigma, che nello sviluppo del pensiero, l'esempio 
ha finito per essere uno dei dispositivi del ragionamento analogico, fondato sulla esemplarità del simile.

Il terzo analogico (o paradigma) comprende i caratteri degli elementi del discorso: essi diventano in esso indiscernibili. Nel paradigma, grazie alla generalizzazione, gli elementi si neutralizzano ${ }^{75}$ - è infatti pressoché impossibile separare il terzo analogico, il paradigma, dalla sua paradigmaticità, dalla capacità di valere per tutti, dall'essere caso singolo ed al contempo tutti i casi di quel genere ${ }^{76}$. II paradigma, secondo Victor Goldschmidt ${ }^{77}$, pur essendo un fenomeno singolare, contiene la forma ${ }^{78}$ che si tratta di definire, l'éidos $(\varepsilon \tilde{\delta} \delta \circ \varsigma)^{79}$. Se la forma è éidos, l'immagine è eidolon ( $\left.\varepsilon i \delta \omega \lambda o v\right)$, ed entrambi vanno letti insieme all'aggettivo idios ('̈ठı૬ৎ), che significa proprio.

II paradigma non è semplicemente elemento sensibile, precipitato della realtà, evento; è questo e, insieme, il rapporto tra l'elemento concreto e la sua definizione - per questo può stare al posto di una descrizione mancante, come nel caso della lacuna del diritto. Così come accade nella reminiscenza platonica (luogo esemplare della conoscenza), il particolare è messo in relazione con l'universale, esattamente come un fenomeno sensibile viene messo in relazione con uno non- sensibile, e così può avvenire un ri-conoscimento.

Si è visto che l'analogia può essere scomposta in giudizio, argomento, concetto. Quest'ultimo in tedesco è Begriff, e contiene la radice di greifen, afferrare. Dunque il concetto analogico è anche ciò che si riesce ad afferrare, è il comprendibile nel senso innanzitutto di prendibile. II movimento del prendere va considerato insieme al tema della forma. Come si legge nel De anima ${ }^{80}$, l'anima è proprio come la mano, poiché nell'anima, come nella mano, c'è la forma della pietra e non la pietra ${ }^{81}$. Come si è sostenuto, il paradeigma ha anche e soprattutto una funzione rappresentativa ${ }^{82}$, ed ecco allora che alcune tracce si ricompongono. L'esempio è un elemento rappresentativo fra molti che contiene i tratti rilevanti che distinguono la categoria esemplificata dalle altre. Ad esso compete dunque anche un'operazione di differenziazione. Cosa fa, d'altronde, il paradigma di un verbo? Traccia i caratteri fondamentali designando gli elementi di distaccamento dalla generalità ${ }^{83}$.

Nella Kritik der reinen Vernunft Kant afferma che ogni tipo di schematismo è un mistero che possiamo afferrare solo pragmaticamente e fenomenologicamente- ciò che prova che lo schema, così come le forme di generalizzazione ${ }^{84}$ di cui fanno uso il diritto e gli altri campi della scienza, sono il luogo del contatto fra pragma e definizioni universali. Kant ritiene infatti che si conosca l'immagine come prodotto empirico della immaginazione produttiva e lo schema come monogramma della immaginazione pura apriori, che rende possibili le immagini. Quando sostiene ciò, si sta in fondo appellando all'esemplarismo ${ }^{85}$.

Usando l'analogia, il giudice non constata semplicemente una somiglianza, come è stato più volte sostenuto, ma la produce attraverso un'operazione a più passaggi. Il paradigma è già dato? Esiste già e deve soltanto essere scoperto? Oppure è oggetto di una produzione? A cominciare 
da Platone ${ }^{86}$ e arrivando fino allo storico francese Yan Thomas, è stato messo in rilievo il carattere poetico del giudizio analogico, il quale si produce secondo necessità, ma che (qui si svela la trama) a suo modo è già insito nella natura degli elementi che operano in un contesto, nel nostro caso all'interno di un ordinamento giuridico, siano questi norme o di decisioni precedenti che vengono prese come esempio di una regola ${ }^{87}$.

La domanda conclusiva è dunque: come e perché si origina lo "stare al posto di una definizione" del ragionamento analogico? Se la forza dell'esemplarità è già insita nell'esempio, allora è impossibile decidere sulla sua origine. La funzione crea ogni volta una nuova origine, come accade alla norma pensata per un caso che diventa norma anche per un altro. II problema della origine va letto in relazione al tema della definizione: la loro interferenza mostra fino in fondo le radici della questione. II caso esemplare che viene usato analogicamente diviene tale perché sospende e, insieme, espone la sua appartenenza al sistema: per questo non è possibile dire esattamente dove abbia origine la sua esemplarità e perchè esso si erga a paradeigma. Nemmeno è possibile dirlo definitivamente.

Per questo non basta guardare l'analogia e l'esempio soltanto con la lente della logica, perché questa non può rispondere a tutte le domande. Nella analogia vige il principio di contraddizione inclusa, di tensione e di dipolarità; nessuna contraddizione è mai decisiva, altrimenti l'inferenza sarebbe rallentata. L'identità elementare della logica, nel campo ana-logico diviene funzionale. Alla discretezza, per cui gli elementi sono connessi solo da relazioni "esterne", il diritto (in quanto si serve di esempi per risolvere complessità) sostituisce il principio di continuità. Le relazioni sono interne e, soprattutto, fra ogni due elementi, per quanto prossimi siano, c'è sempre posto per un terzo.

Thomas Kuhn ${ }^{88}$, fra gli elementi costutitivi di una matrice disciplinare, ha elencato: le generalizzazioni simboliche, ovvero le componenti formali o facilmente formalizzabili; i modelli, che "svolgerebbero una funzione euristica", fornendo alla comunità scientifica "le analogie preferite", o addirittura "un'ontologia"; gli esemplari, che "sono concrete soluzioni di problemi" accettate da una determinata comunità scientifica ${ }^{89}$.

\section{CONCLUSIONI}

In questo saggio ho tentato di dimostrare che la realtà è il luogo della massima connessione fra i fatti e le norme. Voglio concludere che non è possibile distinguere, in un paradeigma, una arché. Ogni fenomeno, ogni norma (in ogni ordinamento) è, intrinsecamente, capace di essere origine, capace di stare al posto di. La funzionalità di un paradigma in un contesto determinato è il risultato di una interferenza fra diacronia e sincronia. Heidegger "in Unterwegs zur Sprache ${ }^{90}$ mette in relazione la mostruosità e il mostrare proprio del segno. Mostruoso è ciò che si mostra e, mostrandosi (Beispiel), lo fa in modo esemplare (Exempel)- né può farlo altrimenti, non ha scelta: la radice di 
Muster $^{91}$ è la stessa di monstre, ciò che si mostra e ciò che mostra oltre se stesso"92. II paradigma rappresenta perciò fino in fondo la ambivalenza costitutiva dell'arché, che è una delle chiavi fondamentali per comprendere gli ordinamenti giuridici contemporanei.

\title{
PER ESEMPIO
}

\begin{abstract}
Analogies, paradigms and exemplars share a common and complex history. Aristotle actually used the term "paradigm" to describe what philosophy later came to call "analogy". All three of these argumentative tools are located in between theory and practice. The article argues that the debate about whether they are more theoretical or more practical - reminiscent of the question discussed in the context of legal realism whether law consists of (practical) experience or (theoretic) rules - is not helpful as one cannot properly locate the origin of paradigmaticity.
\end{abstract}

Key Words: Analogies. These Argumentative. Exemplars. Paradigm.

\section{NOTAS}

1 La dottoressa Angela Condello é dottoranda in Filosofia del diritto presso l'università Roma III, dove lavora come assistente del Prof. Eligio Resta. Contemporanemante è tutor presso I'Università Telematica Internazionale Uninettuno. Attualmente è visiting scholar all'istituto Max Planck Institut für ausländisches öffentliches Recht und Völkerrecht di Heidelberg.

2 Da un adagio giustinianeo in Institutiones, libro II $(7,3)$.

3 Norberto Bobbio, L'analogia nella logica del diritto (1938), a cura di Paolo di Lucia e Luigi Ferrajoli, Giuffrè, Milano (2006).

4 Come lo mostra, per fare un esempio, la frequenza con cui viene citato il termine "analogy" nelle discussioni della Supreme Court USA: 09-158, Magwood vs. Patterson, Walder, et al., (2010); 06-923, Metropolitan Life, Insurance Company et al. vs. Wanda Glenn (2008); 04759, United States vs. Joseph Olson, et al. (2005); 00-758, United States Postal Service vs. Maria A. Gregory (2001); 02-1389, United States vs. Abel Cosmo Galletti et al. (2004); 0610119, Allen Snyder vs. Louisiana (2007); 01-1269, City of Cuyahoga Falls, Ohio, et al. vs. Buckeye Community Hope Foundation, et al. (2003); 04-278, Town of Castle Rock, Colorado vs. Jessica Gonzales et al. (2005); 07-1428, Frank Ricci, et al. vs. John Destefano et al. (2009); 06-313, Don Roper (Superintendent), Potosi correctional center vs. William Weaver (2007); 06-1431, Cbocs West, inc. vs. Hedrick G. Humphries (2008); 05-416, Jeanne S. Woodford, et al. vs. Viet Mike NGO (2006); 00-799, City of Los Angeles vs. Alameda Books, inc. et al. (2001), in cui viene usata l'espressione bad analogy; 06-6330, Derrick Kimbrough vs. United States (2007), dove invece troviamo l'espressione better analogy; 01-682, Kay Barnes, etc., et al. vs. Jeffrey Gorman (2002), in cui un giudice, per argomentare, si serve della locuzione closest analogy, suggerendo una possibile gradazione della portata esemplare del contract; 05-1126, Bell Atlantic et al. vs. William Twombly et al. (2006), dove la declinazione è exact analogy, come se alcune analogie fossero più esatte di altre; 01-455, Franconia Associates et al. vs. United States et al. (2002), in cui nella discussione compare l'espressione quick analogy; 00-1406, Chevron U.S.A. inc. vs. Mario Echazabal (2002), dove si legge: "you have to push beyond the analogy"; 03-923, Illinois vs. Roy I. Caballes. (2004)- in cui nella argomentazione si fa riferimento alla bizzarra "duck analogy".

5 Rhetorica, I, ii, 1357b (25-30); Analitica priora, II, xxiv, 69a (13-16): "E' dunque evidente che l'argomentazione fondata sull'esempio non passa dalla parte al tutto, nè dal tutto alla parte, 
ma procede dalla parte alla parte, quando cioè entrambe le parti siano subordiate ad una medesima nozione, ed una delle parti risulti nota. Tale modo di argomentare differisce poi dall'induzione, in quanto quest'ultima [...] parte da una totalità di oggetti indivisibili e prova in seguito l'appartenenza dell'estremo maggiore al medio, senza connettere il sillogismo all'estremo minore, mentre l'argomentazione fondata sull'esempio opera questo collegamento, ed al tempo stesso non conduce la prova sulla base di una totalità di oggetti indivisibili".

6 Enzo Melandri, La linea e il circolo. Studio logico-filosofico sullanalogia (1968), Quodlibet, Macerata (2004), p. 318-319.

7 Nella tradizione cristiana, l'esempio si è invece soprattutto diffuso in relazione alle vite dei santi come esperienze e percorsi da seguire; l'esempio di vita era chiamato conversatio ed esso veniva additato come modello di riferimento, che aveva anche una forte valenza retorica. Modello di condotta e modello di convincimento sono diventati così, in questa prospettiva, due campi difficilmente distinguibili.

8 Ciò che avviene quando il giudice europeo decide secondo la analogia che risponde al nome di principle- based, che si distingue dalla rule- based analogy. Si tratta di ciò che già $\mathrm{i}$ giuristi romani avevano risolto con le notissime formule di analogia iuris e analogia legis. La giurisprudenza della European Court of Justice sull'art. 30 del Trattato UE è particolarmente interessante a riguardo, poiché combina le due tipologie di ragionamento: l'art. 30 contiene una norma che è un ottimo esempio della possibile coesistenza fra particolare ed universale nel ragionamento giuridico. II significato della espressione "quantitative restrictions or measures of equivalent effect" può essere compreso propriamente solo in riferimento ad una linea di precedenti, e senza trascurare i principi generali dell'ordinamento europeo. I casi esemplari a riguardo sono Criminal proceedings against Herbert Gilli and Paul Andres, Case 788/79, [1980] E.C.R. 2071 e Rewe-Zentral AG v. Bundesmonopolverwaltung für Branntwein, Case 120/78, [1979] E.C.R. 649 (meglio noto come caso "Cassis de Dijon").

9 Sul rapporto fra esemplificazione e semplificazione, si veda Maurizio Ferraris, Documentalità. Perché è necessario lasciar tracce, Laterza, Roma- Bari (2009), p. 12 (Esemplificare, non semplificare).

10 G. Cornu, Le règne discret de l'analogie, in Mélanges à André Colomer, Litec (1993), p. 129

11 Cfr. Jacques Bouveresse, Prodiges et vertiges de l'analogie, Raison d'agir, Paris (1999).

12 Gilles Deleuze, Spinoza et le problème de l'expression, Quodlibet, Macerata (1999).

13 Da intendersi nel senso della phronesis aristotelica.

14 Arché è un termine greco denso di significato; esso infatto è, allo stesso tempo e insieme, principio e comando, nel senso di un principio che non solo origina, ma che è anche informatore. Lo ha richiamato Eligio Resta in Diritto vivente, Laterza, Roma- Bari (2008).

15 La dottrina giuridica francese è, a tal riguardo, ricca di suggerimenti. Per esempio, Sthépane Rials, in Le juge administratif français et la technique du standard, Thèse doctorale, Paris II, (1978). L'attenzione all'uso di schemi, modelli, esemplari e più generalmente a riferimenti è aumentata in Francia soprattutto dopo il cosiddetto tournant linguistique come nuovo corso dell'approccio al diritto. A proposito un autore fondamentale è Olivier Cayla, insieme con Paul Amselek; si veda Théorie des actes de Langage, Ethique et Droit, Presses Universitaires de France, con interventi di Jacques Bouveresse, Gérard Cornu, Christophe Grzegorczyk, D. Neil MacCormick, Paul Ricoeur (1966). Lo spunto offerto dalla dottrina francese è di particolare rilievo nel contesto più ampio di questo intervento se si considera che uno dei termini più ricorrenti è, a proposito dello studio critico del diritto e dei suoi dispositivi, pratiques. Pratiche che, sovente, sono studiate all'interno del terrain.

16 E' interessante notare che la forza della analogia ha conosciuto, tanto nella letteratura filosofica quanto in quella giuridica, numerose declinazioni. Mi riferisco in particolare alla dottrina di Common Law, e al contributo di Scott Brewer, Exemplary Reasoning: Semantics, Pragmatics, and the Rational Force of Legal Argument by Analogy, 109 Harvard Law Review 923 (1996). La tesi di Brewer è che la forza della analogia sia principalmente attribuibile al fatto che la struttura logica di questo argomento si basi su esempi appropriati alle regole cui si riferiscono, in una relazione di equilibrio riflessivo. Gerard Cornu l'ha invece definita force tranquille (Le règne discret de l'analogie, 1993): in un sistema -il diritto- che tende alla esaustività e alla certezza, l'analogia attende "vigilante et marginale; [...] elle sert 
de force cachée, de force intrinsèque ou "spirituelle", au rang des sources réelles du droit mais dans une position de réserve".

17 Melandri ristabilisce un equilibrio fra i due concetti chiamando l'esempio argomento analogico: "Procedendo dunque con ordine, esaminiamo in primo luogo il valore dell'esempio come argomento analogico", ibidem, p. 320.

18 Maurizio Ferraris, Estetica razionale (1997), Raffaello Cortina Editore, Milano, ultima edizione (2011), p.311.

19 A proposito della Mostruosità dell'esempio si veda ancora Ferraris (2011), p. 311 (e ss.). Nella collocazione del ruolo e della funzione svolti dall'esempio l'autore osserva la presenza di un atteggiamento che ripete essenzialmente le ragioni della distinzione tra estetica e logica: gli esempi possono essere nocivi, distrendo dalla regola e privilegiando il caso, e magari inducendo anche a generalizzazioni indebite. Si tratta della tesi kantiana secondo cui lo schema è una regola e non una immagine.

20 Immanuel Kant, Grundlegung zur Metaphysik der Sitten (1785), Göttingen (2004).

21 Wolff, Christian, Philosophia rationalis, sive logica, § 40 (1728).

22 Mi riferisco alla nota frase di Justice Oliver Wendell Holmes "The life of the law has not been logic, it has been experience".

23 Prendiamo ad esempio Roscoe Pound: The life of the law: reason or exeperience?, in Tort and Medical Yearbook, 611 (1961), Justice according to law, 13 Columbia Law Review 696 (1913), The spirit of the Common Law, 18 Green Bag 17 (1906). Ancora prima se ne era occupato Justice Holmes; per un quadro completo della sua teoria, Holmes, Oliver Wendell The Collected Works of Justice Holmes (S. Novick, ed.), Chicago: University of Chicago Press, (1995).

24 Immanuel Kant, Kritik der Urteilskraft (1790), Frankfurt, Surkhamp Verlag, (1974).

25 Sebbene Kant sia diffidente nei confronti dell'esempio in quanto tale, e preferisca lo schemase non fosse che lo schema è, a modo suo, esemplare.

26 Se il fine ultimo del diritto è garantire uguaglianza, anche -e soprattutto- nelle differenze, è dunque naturale che la comparazione con schemi di riferimento sia essenziale nel ragionamento del giurista. L'uguaglianza è il luogo della comparazione. Tutta la storia delle politiche del diritto è attraversata dalla questione del trattamento uguale di situazioni uguali e il trattamento diverso di situazioni diverse. Per la riflessione sul problema della uguaglianza, sulla sua origine e sul suo statuto attuale nelle società contemporanee, i riferimenti sono senz'altro John Rawls, A theory of Justice, Belknap Press of Harvard University Press (1999), Justice as Fairness: a restatement, Harvard University Press, (2001) e Amartya Sen, The idea of justice, Allen Lane (2009). Una indagine sull'uso della analogia nella giurisprudenza costituzionale italiana dal 1962 fino a tutto il 2009 conferma che analogia, paradeigma, ed uguaglianza sono fortemente connessi al tema della uguaglianza (Corte Cost. 448/2008, 154/1976, 227/75 , 112/68, 255/82, 276/93, 173/1991, 72/1964, 208/1975, 39/1971, 208/1975, 440/1992, 82/1973, 3/1969, 246/1983, 143/1969, 40/1962, 214/1972, 127/1996, 12/1970, 286/1993, 46/1993, 226/1976, 144/1972).

27 Per uno sguardo sull'attuale dottrina sul precedent nei sistemi di Common Law: F. Schauer, Precedent 39 Stanford Law Review 571; N. Duxbury, The Nature and Authority of Precedent, Cambridge University Press, Cambridge (2008); Neil MacCormick, Rhetoric and the Rule of Law, Oxford Univ. Press, Oxford (2005); L.L. Weinreb, Legal Reason: The Use of Analogy in Legal Argument, Cambridge Univ. Press, Cambridge (2005); Cass Sunstein, On Analogical Reasoning, 106 Harvard Law Review 741 (1993); Scott Brewer, Exemplary Reasoning: Semantics, Pragmatics, and the Rational Force of Legal Argument by Analogy 109 Harvard Law Review 923 (1996).

28 La coppia singolare/universale ha proporzionalità di riferimento con un'altra coppia già menzionata: esclusione/inclusione. La logica universale tende infatti ad includere, mentre nelle logiche singolari prevale l'idea della esclusione.

29 Cfr. Ferraris, Estetica razionale (2011), p. 315.

30 Nella definizione luhmanniana di organizzazione, la generalizzazione congruente delle aspettative rappresenta il fondamentale criterio di riduzione delle aspettative per i sistemi sociali complessi. La generalizzazione congruente caratterizza la struttura normativa di tutti i sistemi sociali della società (cioè delle collettività politiche), in quanto essa definisce secondo Luhmann- anche il diritto. Cfr. Niklas Luhmann, Sociologia del diritto (1972), Bari (1983), p. 105.

31 Kant, Kritik der reinen Vernunft (1787), Meiner Verlag, Hamburg (1998). 
32 Jürgen Habermas, Faktizität und Geltung. Beiträge zur Diskurstheorie des Rechts und des demokratischen Rechtsstaats, Surkamp Verlag, Frankfurt (1998).

33 Sia nella Farbenlehre che nella Metamorphose der Planzen, cfr. Naturwissenschaftliche Schriften, a cura di Ernst Beutler, I-II, in Gedenkausgabe der Werke, Briefe und Gespräche, XVI-XVII, Artemis- Verlag, Zurich (1949-52). Goethe spesso mette a confronto il suo metodo con quello che procede per "casi particolari e rubriche generali, opinioni e ipotesi".

34 Walter Benjamin, Ursprung des deutschen Trauerspiels, in Gesammelte Schriften, a cura di Rolf Tiedemann e Hermann Schweppenhäuser, Surkamp, Frankfurt am Main (1974).

35 Sull'arché come principio e comando allo stesso tempo: Eligio Resta, Diritto vivente, Laterza, Roma- bari (2008).

36 Per cui Urphänomenon è fenomeno originario in quanto precedente, superiore, anteriore e irraggiungibile.

37 Johann Wolfgang von Goethe, Der Versuch als Vermittler von Objekt und Subjekt (1792), in Gedenkausgabe der Werke, Briefe und Gespräche, XVI-XVII, Artemis- Verlag, Zurich (194952), p. 852.

38 ibidem, p. 851-52. Nelle Maximen und Reflexionen, inoltre, Goethe ne compendia ancora di più la natura: "Il fenomeno originario: ideale in quanto è l'ultimo conoscibile/ reale in quanto conosciuto/ simbolico perché abbraccia tutti i casi: identico in tutto i casi" (p. 693).

39 Maurizio Ferraris, Estetica razionale (ult. ed., 2011), p. 311.

40 cfr. Kant e l'esemplarità dell'esempio, M. Ferraris, in Filosofia '94, a cura di Gianni Vattimo, Laterza, Roma-Bari (1995).

${ }^{41}$ Ciò che è avvenuto spesso nella giurisprudenza amministrativa francese; si veda Stéphane Rials, Le juge administratif français et la technique du standard, Thèse doctorale, Paris II, (1978).

42 Il tema del canone è presente nel diritto, nelle arti figurative e nella musica.

43 Questo termine richiama il dibattito americano intorno alla questione: il giudice "crea" diritto o il giudice "scopre" diritto? Jerome Frank, giurista americano vissuto a cavallo fra diciannovesimo e ventesimo secolo, ha lavorato molto a riguardo. Si veda Law and the Modern Mind (1930), Peter Smith Pub. Inc. (1985).

$44 \mathrm{La}$ analogia è intrinsecamente legata al tema della natura delle cose, come lo dimostra la monografia di Arthur Kaufmann, Analogie und Natur der Sache (1965), 2. Auflage (1982). II legame fra le cose, i loro nomi, e le differenti somiglianze che ne possono costituire le relazioni è anche un noto tema foucaultiano: lo troviamo in Les mots et les choses (Paris, Gallimard, 1966), che in L'archéologie du savoir (Paris, Gallimard, 1969). Nel primo caso mi riferisco alla sezione dedicata alle somiglianza: Foucault ne elenca quattro tipi: convenientia, aemulatio, analogia e simpatia, e sostiene che fino alla fine del XVI secolo, esse hanno avuto un ruolo fondamentale nello sviluppo della conoscenza. In L'archéologie du savoir faccio riferimento in particolare al capitolo intitolato "La description archéologique", in cui si trovano passaggi sulla relazione fra l'original et le régulier (p.184) e su les faits compratifs (p. 205).

45 M. Ferraris, Documentalità. Perchè è necessario lasciar tracce, Laterza, Roma- Bari (2009), p. 14.

46 Il tema del tipo (in greco tumos, typos) è molto importante per il diritto, così come anche quello del modo, del modello, e dell'esempio, naturalmente.

47 Kant, Kritik der Urteilskraft, Surkamp Verlag, Frankfurt, p. 349. Allo stesso modo è rilevante il §18, Was die Modalität eines Geschmackurteils sei, in particolare nel passaggio in cui Kant lega la regola generale all'esempio: "Urteil, was wie Beispiel einer allgemeinen Regel, die man nicht angeben kann, angesehen wird", p. 156.

48 Corte Costituzionale e Corte di Cassazione. In particolare la ricerca nel materiale giurisprudenziale di Cassazione ha dimostrato che il dispositivo è usato con maggiore frequenza nelle controversie in materia di diritto del lavoro, diritto sindacale e diritto tributario.

49 Nella più recente giurisprudenza della Supreme Court of England: [2010] UKSC 20, R. (on the application of Sainsbury's Supermarkets Ltd) vs. Wolverhampton City Council and another; [2010] UKSC 20 Agbaje vs. Akinnoye-Agbaje (FC); [2010] UKSC 1 Her Majesty's Treasury vs. Mohammed Jabar Ahmed and others (FC).

50 Declinato talora con un attributo, come per esempio "a useful analogy" ([2010] UKSC 20): ciò che rende perfettamente la funzione pragmatica di questo ragionamento; la analogia ed il 
suo utlizzo non possono essere disgiunti dal concetto aristotelico di phronesis, la saggezza pratica, che rimanda al dibattito sul ruolo della analogia fra i giuristi romani, in particolare al contrasto fra Giuliano e Giustiniano. A tal proposito si veda Letizia Vacca, Metodo casistico e sistema prudenziale, Cedam, Padova (2006). Non è un caso che la phronesis aristotelica sia tradotta in latino con prudentia.

51 Cfr. Klaus Peter Berger, The Creeping Codification of the lex mercatoria, Kluwer Law International, The Hague (1999).

52 Karl Popper, Logik der Forschung, Mohr Siebeck; Auflage: 10. verb. u. verm. A. (2005).

$53 \mathrm{Si}$ veda Annales. Histoire, sciences sociales, vol. 62 (1/2007), volume dedicato interamente alle Formes de la généralisation; in particolare, gli articoli di Jean- Luis Fabiani, La généralisation dans les sciences historiques. Obstacle épistémologique ou ambition légitime?, 1 Annales. Histoire, Sciences Sociales 9- 28 (2007), e di Yan Thomas, L'enfant à naître et l'" héritier sien ». Sujet de pouvoir et sujet de vie en droit romain (p. 29).

54 Alcuni storici del diritto, fra cui Yan Thomas, hanno lavorato sulla preganza del cosiddetto cas-limite; Yan Thomas, L'extrême et l'ordinaire. Remarques sur le cas médièval de la communauté disparue, in Penser par cas (2005), pp. 45- 73.

55 Anabasis era una spedizione che dalla costa risaliva verso l'entroterra; katabasis era il percorso inverso. Senofonte (431- 355 a.C.) nella Anabasis descrive la spedizione di un principe persiano, Ciro il Giovane, contro suo fratello.

56 Oliver Wendell Holmes, The Path of the Law, 10 Harvard Law review 457 (1897).

57 "è difficile trovare espresso in poche righe, sotto la specie della chiarezza apparente, un simile cumulo di incongruenze. [...] E che dire poi di John Stuart Mill, il quale dopo oltre duemila anni, ripete lo stesso errore? Essendo empirista e non razionalista, egli capovolge il criterio aristotelico di classificazione: l'analogia diventa così la forma primaria d'inferenza, ma solo perchè in ultima analisi all inference is from particulars to particulars", Melandri, $p$. 318. J.S.Mill, A System of Logic (1843), II, iii, § 4.

58 Qualche decennio fa, il preside della Chicago Law School e Ministro della Giustizia degli Stati Uniti, Edward Levi, ha pubblicato una monografia intitolata An Introduction to Legal Reasoning; in essa desrive un resoconto del ragionamento giuridico dal punto di vista del professionista del diritto. Per Levi, pensare da giuristi non significa applicare ampie strutture di teoria a questioni giuridiche discrete, ma sia piuttosto ragionare per analogia da un gruppo ad un altro di questioni giuridiche concrete. Cass R. Sunstein, anch'egli professore alla Chicago Law School, ha ripreso e sviluppato l'idea di Levi e l'ha tradotta nell'approccio "incompletamente teorizzato" al diritto (Incompletely Theorized Agreements in Constitutional Law, Social Research, 2007).

59 Ronald Dworkin, Justice in Robes, Harvard University Press (2006); si vedano in particolare i capitoli Pragmatism and Law (p. 36), In Praise of Theory (p. 50) e Darwin's new Bulldog (p. 75), in cui critica la posizione di Richard Posner.

60 Edward H. Levi, An Introduction to legal reasoning, I (1949), ("It is important that the mechanism of legal reasoning should not be concealed by [the] pretense...that the law is a system of known rules applied by a judge; the pretense has long been under attack"); allo stesso modo Felix Cohen, The Ethical Basis of Legal Criticism, 41 Yale Law Journal, 201, 215 (1931), ("The confusion arises when we think of a judicial decision as implying a rule from which, given the facts of the case, the decision may be derived...").

${ }^{61}$ Per fare soltanto i nomi più importanti, Charles Sanders Peirce, William James, e John Dewey. A pag. 22 di Pragmatism, James scrive "I think you will practically recognize the two types of mental make up that I mean if I head the columns by the titles 'tender minded' and 'tough-minded' respectively: the tender minded (is) rationalistic (going by 'principles'), intellettualistic, idealistic, optimistic, religious, free- willist, monistic, dogmatical [...]; the tough-minded (is) empiricist (going by 'facts'), sensationalistic, materialistic, pessimistic, irreligious, fatalistic, pluralistic, sceptical'.

62 Dworkin ha respinto con forza questa posizione, e lo ha fatto richiamando Kant: "l'analogiaha detto- senza teoria è cieca". II ragionamento analogico, è -secondo Dworkin- un modo di affermare una conclusione, ed è la teoria a fare la gran parte del lavoro.

63 In questo dibattito intorno alla vera natura delle decisioni giudiziarie va considerato il contributo di Richard Posner, in particolare con il suo testo How Judges think (Harvard University Press, 2007). A proposito dell'uso di ragionamenti come quello analogico nel diritto, Posner distingue due principali linee teoriche: da un lato, i cosiddetti legalists, come 
Ronald Dworkin, dall'altro i pragmatisti- e in particolare i neopragmatisti, come Richard Rorty.

64 Richard A. Posner, How Judges Think, Reprint Edition Harvard University Press (2010). In particolare, mi riferisco al capitolo Is Pragmatic Adjudication Inescapable?, p. 230.

65 "The life of the law has not been logic; it has been experience. The felt necessities of the time, the prevalent moral and political theories, intuitions of public policy, avowed and unconscious, even the prejudices which judges share with their fellow-men, have had a good deal more to do than the syllogism in determining the rules by which men should be governed", in The Common Law 1 (1881).

66 Sulla adjudication ha lavorato, fra gli altri, uno fra i maggiori esponenti del movimento dei Critical Legal Studies, Duncan Kennedy (A Critique of Adjudication [fin de siècle], Harvard University Press, 1997).

67 Maurizio Ferraris, Estetica razionale (1997), Raffaello Cortina Editore, Milano (2011), p. 302 e ss.

68 Michel Foucault, L’archéologie du savoir, éd. Gallimard, Paris (1969), p.184.

69 Enzo Melandri, ibidem.

70 Cit. pag. 546.

71 Ibidem, p. 546.

72 Come nella poesia di Wallace Stevens intitolata Description without place (1945):

73 "[1] It is possibile that to seem - it is to be/As the sun is something seeming and it is/The sun is an example. What it seems/lt is and in such seeming all things are/Thus things are like a seeming of the sun/Or like a seeming of the moon or night/ Or sleep. It was a queen that made it seem / By the illustrious nothing of her name. / Her green mind made the world around her green./ The queen is an example... This green queen / In the seeming of the summer of her sun/ By her own seeming made the summer change./ In the golden vacancy she came, and comes,/ And seems to be on the saying of her name./ Here time becomes again, as it became, / The crown and week-day coronal of her fame./ [2] Such seemings are the actual ones: the way/ Things look each day, each morning, or the style/ Peculiar to the queen, this queen or that,/ the lesser seeming original in the blind"

74 Il diritto ridefinisce constantemente ciò che è rilevante; sul tema della rilevanza nelle scienze sociali, Alfred Schutz, Das Problem der Relevanz, Suhrkamp, Frankfurt am Main (1982).

75 E' ciò a cui allude Aristotele quando, parlando della giustizia distributiva, introduce la analogia (Etica Nicomachea, V, vi, II3la29-b24).

76 Cfr. Giorgio Agamben, Signatura rerum, Che cos'è un paradigma? (2008), p. 22.

77 Annales. Histoire, sciences sociales, vol. 62 (1/2007), sulle Formes de la généralisation.

78 V. Goldschmidt, Le paradigme dans la dialectique platonicienne, Vrin, Paris (1947).

79 II problema della forma è assai ricorrente tanto nella filosofia quanto nel diritto. Cfr. G.W.F. Hegel, Grundlinien der Philosophie des Rechts. Naturrecht und Staatswissenschaft, Herausgegeben und eingeleitet von Helmut Reichelt, Ullstein, Frankfurt am Main (1972). Per Hegel la scienza filosofica del diritto ha per oggetto l'Idea di diritto, il concetto e la realizzazione del concetto di diritto. Cioè la forma e la figurazione dell'Idea di diritto. La forma del concetto o idea di diritto è la sua forma logica- così come noi ora la esponiamo e la concepiamo. La figurazione dell'ldea di diritto è la realizzazione del diritto nella realtà storica, il suo processo di realizzazione. Potremmo dunque dire che la figurazione è una idea inserita in un contesto. L'idea-concetto di diritto contiene entrambe le determinazioni (razionale e reale). Per Idea si intende proprio l'unità di concetto e realtà. L'Idea è quel concetto che si conferisce la realtà da se stessa, ossia è quel procedere razionale che dà senso alla realtà; ricomprende in sé la realtà che permane e che non è transeunte. Questa corrispondenza fra realtà e concetto (nell'ldea) è la stessa corrispondenza fra forma semplicemente concettuale e figurazione del concetto nel corso della sua realizzazione. "[...] lo sviluppo da fondamenti storici, infatti, non va scambiato con lo sviluppo dal Concetto, e alla spiegazione e giustificazione storica non bisogna dare il significato di una giustificazione valida in sé e per sé", pag. 79.

80 In max Weber il tipo ideale o Idealtipo è lo strumento specifico di lavoro dello scienziato sociale. II tipo ideale non è la rappresentazione fedele di un dato reale, ma è idealizzazione, in quanto concetto costruito mediante l'accentuazione di determinati elementi della realtà empirica: "Nella sua purezza concettuale questo quadro non può essere mai rintracciato empiricamente nella realtà; esso è un'utopia, e al lavoro storico si presenta il compito di 
constatare in ogni caso singolo la maggiore o minore distanza della realtà da quel quadro ideale" (II metodo delle scienze storico-sociali, Torino, 1958, p. 108 (titolo originale: Gesammelte Aufsätze zur Wissenschaftslehre, 1922).

81 432a (2-3).

$82 \mathrm{Da}$ questa tradizione deriva il frequente ricorso filosofico alla mano come strumento "universale" (per esempio in Cartesio, Discours de la méthode, AT, VI: 57), o come strumento "assoluto", fino ad arrivare ad Heidegger e al suo elogio della mano come proprio dell'uomo, in Was heißt Denken?, in Gesamtausgabe. Qui Hedegger si propone di dimostrare che ogni pensiero, ogni definizione, è un manufatto. L'anima è, sia in Platone, che in Aristotele, che in Heidegger, il luogo della immaginazione. Ciò che rimane nella immaginaizone (ciò che Ferraris chiama la traccia) assicura la temporalità. Dunque, non possiamo parlare di forma senza fare riferimento alla figurazione.

83 II Purgatorio di Dante, in particolare fra i canti IX e XX, è ricchissimo di exempla. Dante nel Canto XXI vede disegnati molti esempi di punizione di superbi ("Come/perché di lor memoria sia/sovra i sepolti le tombe terragne portan segnato quel ch'elli eran prima/Onde lì molte volte si ripiagne per la puntura de la rimembranza, che solo à pii dà de le calcagne"). Nel V girone, Dante e Virgilio odono un'anima che ricorda loro esempi di onestà e santa povertà e di nobile larghezza (Ugo Capeto).

${ }^{84}$ Infatti è particolarmente importante nel caso di verbi irregolari.

85 A tale proposito non si deve trascurare la rilevanza dei processi di generalizzazione fra storia e teoria del diritto, come ha raccontato Yan Thomas in L'extrême et l'ordinaire. Remarques sur le cas médièval de la communauté disparue, in Penser par cas (2005), p. 45- 73.

86 Cfr. Ferraris (2011), p. 314.

87 Platone, Politico (278 c, 4), "paradeigmatos [...] génesis; paradeigmata [...] gignomena".

88 Ciò che, come è ben noto, accade negli ordinamenti di Common law, nei quali tra l'altro (è bene precisarlo) non è prevista la analogia per gli Statutes. Si tratta in ogni caso di un ragionamento che da una somiglianza conduce ad una inferenza.

89 Thomas Kuhn, Nuove riflessioni sui paradigmi (1974) e The Structure of Scientific Revolutions (1962). Kuhn sembrerebbe rinvenire nelle strategie d'uso degli esemplari delle vere e proprie prassi, costitutive ed a priori, di categorizzazione di quell'esperienza cui si farà riferimento per il controllo delle stesse teorie. E questo lo porta ad interpretare in chiave semantica e categoriale l'uso degli esemplari. Se il sistema ipotetico deduttivo deve essere sostituito da una più ampia matrice disciplinare, al cui interno assumono principale importanza gli esemplari, saranno questi, per Kuhn, a svolgere la funzione di canale di entrata del significato empirico delle teorie. Poichè, storicamente e geneticamente, la pratica di problem-solving tramite riduzione analogica gioca una parte significativa nel ruolo che viene abitualmente affidato dagli epistemologi ipotetico-deduttivisti alle regole di corrispondenza, Kuhn ritiene opportuno sostituire dette regole con una strategia di riduzione tramite esemplari, cioè esempi-standard condivisi da una determinata comunità scientifica attraverso cui "le generalizzazioni simboliche" acquistano il loro significato e contenuto empirico che proviene loro " tanto dal basso quanto dall'alto".

90 Non si può lasciare fuori da questo quadro Karl R. Popper, che in Scienza e Filosofia (trad. it. di M. Trinchero, Einaudi, Torio, 1969, p.150), dice " [...] tendiamo a scoprire nuovi mondi dietro il mondo dell'esperienza ordinaria, mondi come, ad esempio, un mondo microscopico o submicroscopico; come, ad esempio, un mondo non-euclideo, un mondo popolato di forze invisibili: forze gravitazionali, chimiche, elettriche e nucleari, alcune delle quali, forse, sono riducibili ad altre, mentre altre non lo sono."

91 M. Heidegger, Unterwegs zur Sprache, XIV ed. Klett- Cotta, Stuttgart (2007).

92 Che in tedesco significa campione, disegno, modello.

93 Cit., Maurizio Ferraris, Estetica razionale (ed. 2011), p. 318.

Recebido para publicação: 25/07/2011

Aceito para publicação: 25/11/2011 